transcription polymerase chain reaction (rRT-PCR), for a prevalence of $8.3 \%$, which was much higher than the frequency of COVID-19 in general population ( 2 per 10,000) during this 4 week period.

One hypothesis is that aging is a condition associated with inflammation, whereas children might have an immature anti-inflammatory response. Possibly, therefore, an increased inflammatory reaction is expected in adult subjects compared to children. ${ }^{7}$

Not only the pediatricians but also other healthcare professionals are at a higher risk of infection with COVID-19. ${ }^{8}$ A report from Italy revealed that $\sim 20 \%$ of healthcare professionals had become infected. ${ }^{9}$ Healthcare professionals are at the frontline of fighting COVID-19 in hospitals, where they are in contact with visiting patients and their parents who are potentially carriers of SARS$\mathrm{CoV}-2$, even if they are not infected. Indeed these healthcare professionals are under excessive workload pressure and psychological distress during the pandemic, which can lead to caregiver burnout. ${ }^{10}$ Pediatricians, especially those who have children at home, not only have concerns about passing the infection to their children but also about not caring enough for their children during quarantine period, considering school closures and social distancing policies. ${ }^{8}$ Therefore, healthcare systems should be very careful to address the physical and mental health of healthcare professionals. Easy access to personal protective equipment, especially for those who are visiting patients with COVID-19, and psychological support for those who are losing their patients and colleagues, especially for those who cannot see their family members for long periods, are necessary.

Acknowledgments. This letter is dedicated to honoring the memory of our brave fallen doctors and nurses who fought against COVID-19.
Financial support. No financial support was provided relevant to this article.

Conflicts of interest. All authors report no conflicts of interest relevant to this article.

\section{References}

1. Johns Hopkins Coronavirus Resource Center website. https://coronavirus. jhu.edu/map.html. Accessed April 4, 2020.

2. Dong Y, Mo X, Hu Y, et al. Epidemiology of COVID-19 among children in China. Pediatrics 2020 March 16. doi: 10.1542/peds.2020-0702.

3. Cruz A, Zeichner S. COVID-19 in children: initial characterization of the pediatric disease. Pediatrics 2020 March 16. doi: 10.1542/peds.2020-0834.

4. Lu X, Zhang L, Du H, et al. SARS-CoV-2 infection in children. N Engl J Med 2020 Mar 18. doi: 10.1056/NEJMc2005073.

5. Wu Z, McGoogan JM. Characteristics of and important lessons from the coronavirus disease 2019 (COVID-19) outbreak in China: summary of a report of 72314 cases from the Chinese Center for Disease Control and Prevention. JAMA 2020 Feb 24. doi: 10.1001/jama.2020.2648.

6. COVID-19 daily epidemiology journal. Iranian Ministry of Health and Medical Education website. http://corona.behdasht.gov.ir/files/sitel/files/Factsheet_4. _26.12_-_En.pdf. Published March 16, 2020. Accessed April 15, 2020.

7. Saghazadeh A, Rezaei N. Immune-epidemiological parameters of the novel coronavirus-a perspective. Expert Rev Clin Immunol 2020 Apr 1. doi: 10. 1080/1744666X.2020.1750954.

8. Lo D. COVID-19: protecting healthcare workers. Lancet 2020;395(10228): 922. doi: 10.1016/S0140-6736(20)30644-9.

9. Remuzzi A, Remuzzi G. COVID-19 and Italy: what next? Lancet 2020 Mar 13. doi: 10.1016/S0140-6736(20)30627-9.

10. Moazzami B, Razavi-Khorasani N, Dooghaie Moghadam A, Farokhi E, Rezaei N. COVID-19 and telemedicine: immediate action required for maintaining healthcare providers well-being. J Clin Virol 2020 Apr 4. doi: $10.1016 / j . j c v .2020 .104345$.

\title{
An easy protective measure in ophthalmology against medical supply shortage
}

\author{
Fen Tang ${ }^{1,2}$, Guangyi Huang ${ }^{1}$, Wei Huang ${ }^{1}$ and Fan Xu MD ${ }^{1}$ \\ ${ }^{1}$ Department of Ophthalmology, People's Hospital of Guangxi Zhuang Autonomous Region, Nanning, Guangxi, China and ${ }^{2}$ Department of Ophthalmology, \\ Renmin Hospital of Wuhan University, Wuhan, Hubei, China
}

To the Editor-The rapidly spreading pandemic of coronavirus disease 2019 (COVID-19) poses a huge challenge to global public health. Healthcare systems in many countries are facing severe shortages of medical supplies. ${ }^{1-3}$

Healthcare workers in ophthalmology departments are susceptible to cross infection because routine ophthalmic examinations, such as like slit-lamp microscopy, are usually performed in a setting of close doctor-patient contact. ${ }^{4}$ Three ophthalmologists in Wuhan Central Hospital were infected with SARS-CoV-2 and died from severe COVID-19., ${ }^{5,6}$ At the ophthalmic center of Guangxi

Author for correspondence: Fan Xu, E-mail: oph_fan@163.com

Cite this article: Tang F, et al. (2020). An easy protective measure in ophthalmology against medical supply shortage. Infection Control \& Hospital Epidemiology, 41: 1107-1108, https://doi.org/10.1017/ice.2020.136
Province, we have continued to provide medical services for outpatients who need timely services during the COVID-19 pandemic. However, in response to the lack of personal protective equipment (PPE) (eg, face shields and gowns) during the epidemic peak in China, ${ }^{7}$ we have adopted an easy measure to perform slitlamp examinations. After thorough disinfection, we installed a home-made shield at the slit-lamp to separate patients and ophthalmologists. As shown in Figure 1, the protective shield is placed between the chin rest/headrest and the microscope.

The protective shield could prevent contamination of the slitlamp by respiratory droplets. Although the shortage of medical supplies is currently being alleviated in China, we are encouraging the continuation of this easy protective measure to curb cross transmission in ophthalmology and to protect the ophthalmologists on the frontline of the pandemic. 


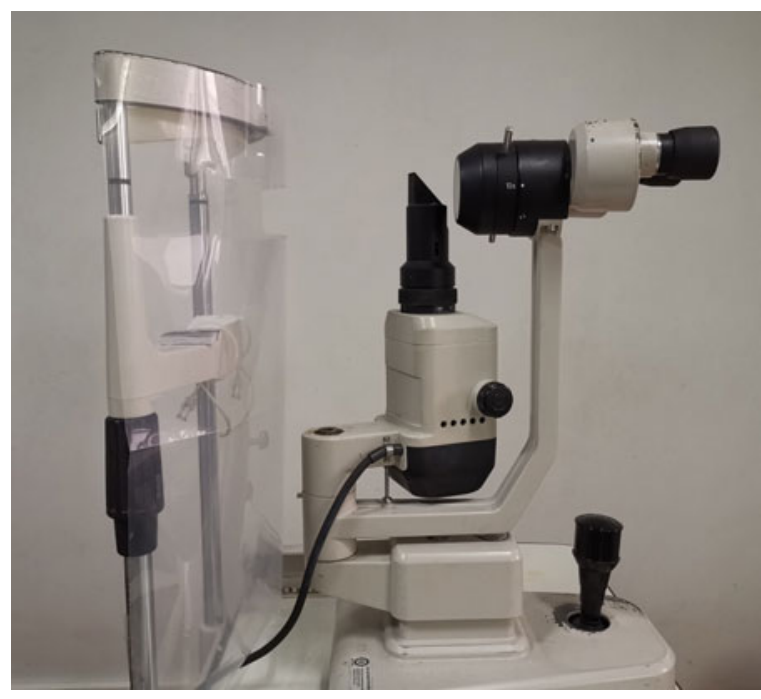

Fig. 1. A transparent shield at the slit lamp.

Acknowledgments.

Financial support. No financial support was provided relevant to this article.
Conflicts of interest. All authors report no conflicts of interest relevant to this article.

\section{References}

1. Namazi I. Sanctions and medical supply shortages in Iran. Wilson Center website. https://www.wilsoncenter.org/publication/sanctions-and-medicalsupply-shortages-iran. Published 2020. Accessed March 28, 2020.

2. Ranney ML, Griffeth V, Jha AK. Critical supply shortages: the need for ventilators and personal protective equipment during the COVID-19 pandemic. N Engl J Med 2020. doi: 10.1056/NEJMp2006141.

3. Barry C. Virus marches on in Italy, Iran, US; medical supplies shrink. Business Insider website. https://www.businessinsider.com/italian-doctor-dies-fromcoronavirus-covid-19-after-warning-low-supplies-2020-3. Published 2020. Accessed March 28, 2020.

4. Seah I, Su X, Lingam G. Revisiting the dangers of the coronavirus in the ophthalmology practice. Eye (Lond) 2020. doi: 10.1038/s41433-020-0790-7.

5. Parrish RK, Stewart MW, Duncan Powers, SL. Ophthalmologists are more than eye doctors-in memoriam, Li Wenliang. Am J Ophthalmol 2020. doi: 10.1016/j.ajo.2020.02.014.

6. Four doctors at Wuhan hospital where coronavirus whistle-blower worked have died of the disease in just over a month. Daily Mail website. https://www.dailymail.co.uk/news/article-8091705/Fourth-colleague-Chinascoronavirus-whistle-blower-doctor-dies-disease.html. Published 2020. Accessed March 28, 2020.

7. The shortage of medical supply in China [in Chinese]. Deutsche Welle website. https://www.dw.com/zh/单日新增确诊数最高-中国医疗物资告急/ a-52273498. Published 2020. Accessed March 28, 2020.

\title{
Practical implementation of COVID-19 patient flags into an antimicrobial stewardship program's prospective review
}

\author{
Ryan W. Stevens PharmD, BCIDP (10), Lynn Estes PharmD and Christina Rivera PharmD, BCPS, AAHIV-P \\ Department of Pharmacy Services, Mayo Clinic, Rochester, Minnesota
}

To the Editor-In March 2020, a call to action was issued for antimicrobial stewardship programs (ASP) to assist in the SARSCoV-2/COVID-19 response. ${ }^{1}$ Specific attention was focused on the common ASP infrastructures of prospective audit, existing partnerships with microbiology laboratories, and experience in stewarding medication resources as justification for ASP involvement. We leveraged our existing Enterprise ASP prospective audit platform to contribute to the response. Here we describe the logic and development of COVID-19 ASP flags, which were rapidly operationalized in the enterprise electronic medical record (EMR).

Our prospective audit system utilizes a longstanding, homegrown flagging system that was converted to function within the EMR (Epic Systems, Verona, WI). ${ }^{2-4}$ It generates a patient list based on a series of "rules" with complex logic incorporating medications and order elements, laboratory values, and microbiology, etc. The system also allows for the documentation of actions taken and provider response. Interventions deemed complete

Author for correspondence: Ryan W. Stevens, 200 1st St. SW Rochester, MN 55905. E-mail: stevens.ryan@mayo.edu

Cite this article: Stevens RW, Estes L, and Rivera C. (2020). Practical implementation of COVID-19 patient flags into an antimicrobial stewardship program's prospective review. Infection Control \& Hospital Epidemiology, 41: 1108-1110, https://doi.org/10.1017/ ice. 2020.133 can be dismissed (ie, removed), and those requiring follow-up can be deferred for later review.

As the burden of COVID-19 patients began to increase, and amid concerns regarding medication shortages, our ASP needed a mechanism to identify patients with a SARS-CoV-2 polymerase chain reaction (PCR) testing performed and/or patients receiving medications in need of careful stewardship. We elected to incorporate both the PCR result and potential COVID-19 therapies into the rule logic because flagging the medications alone would not filter out non-COVID-19 indications, leading to the addition of low value flags or "noise" into the system. We considered flagging only PCR-positive patients, but this approach would fail to identify pending testing or PCR-negative patients who remained on potentially inappropriate medications. Conversely, incorporation of all ordered PCRs would also have contributed a great deal of noise. We developed a hybrid approach by designing 2 flags that identify opportunities for stewardship of medications and confirm infectious diseases (ID) consultation.

The first rule (ASP COVID-19 rule 1) uses logic that identifies inpatients with a negative SARS-CoV-2 PCR test, collected within the previous 7 days, who also have a medication order that may represent "active therapy" (Table 1). Despite the 2 studies by Gautret et $\mathrm{al}^{5,6}$ claiming the benefit of the combination of 